\title{
Approximate method for fast retrieval of particle size distribution in spectral extinction technique
}

\author{
Li Wang, ${ }^{* 1}$ Xiaogang Sun, ${ }^{1}$ and Feng $\mathrm{Li}^{2}$ \\ ${ }^{1}$ Department of Automation Measurement and Control, Harbin Institute of Technology, Harbin 150001, China \\ ${ }^{2}$ School of Electronics Information Engineering, Harbin Institute of Technology, Harbin 150001, China
}

Received November 08, 2011; accepted December 19, 2011; published December 31, 2011

\begin{abstract}
Generalized eikonal approximation (GEA) method, used as an alternative to the rigorous Mie theory in this paper, is introduced for retrieval of the particle size distribution (PSD) in the independent model. To compute the extinction efficiency accurately, the combination of GEA method and Mie theory is adopted, which can not only extend the applicable range of the approximation method but also improve the speed of the whole retrieval. Both simulations and experimental results show the method can be successfully applied to the retrieval of PSD when the refractive index is within the validity range. By using this method, we find the complexity and computation time of the retrieval are significantly reduced, and the memory resources can also be saved effectively. Thus all of these factors make the method more suitable for realization of on-line particle sizing.
\end{abstract}

Fast retrieval of particle size distribution (PSD) is very important in many practical applications. Among the measuring methods, the spectral extinction technique is very promising because it only requires a simple optical layout and a commercial spectrophotometer [1-3].

In the spectral extinction technique, how to calculate the extinction efficiency quickly and accurately is a critical issue which affects the accuracy and rapidity of the whole retrieval. Traditional retrieval practice is based on extinction efficiency calculated by the rigorous Mie theory. However, its calculation is difficult and time consuming, in particular, the computation time is dramatically increased with increasing range or subintervals of the particle diameters [4]. Therefore, it is highly desirable to apply a simple and accurate approximation method to retrieve PSD over a relatively wider applicable range.

In this paper, the generalized eikonal approximation (GEA), used as an alternative to the rigorous Mie theory is introduced for retrieval of PSD in a spectral extinction technique. To compute the extinction efficiency accurately, the combination of Mie theory and GEA method is adopted. Within the framework of a combined approximate method, the accuracy and limitations of retrieval are then investigated in an independent model.

Consider a beam of monochromatic light of intensity $I_{0}$ traveling through a suspension of small particles. In the absence of multiple scattering, the transmitted light

*E-mail: lwanghit@yeah.net intensity $I$ is given by [5]:

$$
\ln \left(\frac{I}{I_{0}}\right)_{\lambda i}=-\frac{3}{2} L N \int_{D_{\min }}^{D_{\max }} \frac{Q_{e x t}\left(\lambda_{i}, m, D\right) f(D)}{D} d D \quad i=1,2, \cdots s
$$

where $\left(I / I_{0}\right)_{\lambda i}$ is the extinction value. $L$ and $N$ are the optical path length and total number of particles, respectively; $s$ is the number of wavelengths; $Q_{\text {ext }}\left(\lambda_{i}, m, D\right)$ is the extinction efficiency; $f(D)$ is the unknown PSD volume frequency distribution function to be retrieved.

The GEA method can be employed to study the light scattering of arbitrarily shaped particles at small scattering angles. The method is to generalize the linear propagator in the eikonal approximation (EA) by adding two adjustable parameters, which compensate for the phase change and edge effect neglected in the EA method [6, 7]. The extinction efficiency for a spherical particle is:

$$
\begin{aligned}
Q_{e x t}(\lambda, m, D)=2 \operatorname{Re}\{ & -i \frac{2}{3} \alpha_{0} \gamma(1-\gamma) \rho \\
& \left.+\alpha_{0} \gamma^{2}\left[1+\frac{2 i}{\rho} e^{i \rho}+\frac{2}{\rho^{2}}\left(1-e^{i \rho}\right)\right]\right\}
\end{aligned}
$$

where $\alpha_{0}=\frac{m+1}{2}-i \frac{3}{8}\left[\frac{1}{x}-\frac{2}{\rho}\left(\frac{a_{1}}{x^{2 / 3}}-\frac{a_{2}}{x^{4 / 3}}\right)\right], x=\frac{\pi D}{\lambda}, \rho=k D(m-1)$, $\gamma=(m+1) /\left(2 \alpha_{0}\right)$. Here $a_{1}=2.0+2.4 i, a_{2}=2.0+6.0 i$ are the coefficients of two edge effect terms which are determined by matching the values of extinction efficiency and imaginary part of scattering amplitude at large size parameter $x$ and at the first maxima [7].

The GEA method considers the phase change and edge effect in the whole diameter region, while the edge effect should be negligible for small particles. In order to reduce errors as well as ensure the rapidity of the whole retrieval, in this paper, the combination of Mie theory and GEA method is applied to calculate the extinction efficiency.

$$
Q_{\text {ext }}(\lambda, m, D)=\left\{\begin{array}{cc}
Q_{\text {Mie }}(\lambda, m, D) & 0 \leq D \leq 1 \\
Q_{G E A}(\lambda, m, D) & D>1
\end{array}\right.
$$

Figure 1 shows a comparison of extinction efficiency calculated by the Mie theory and combined approximate method. From these figures, the extinction efficiency calculated by the combined approximate method contains the correct geometrical optical limit and the right extreme 
points. The maximum relative error of the combined approximate method is significantly reduced compared with the original GEA method.
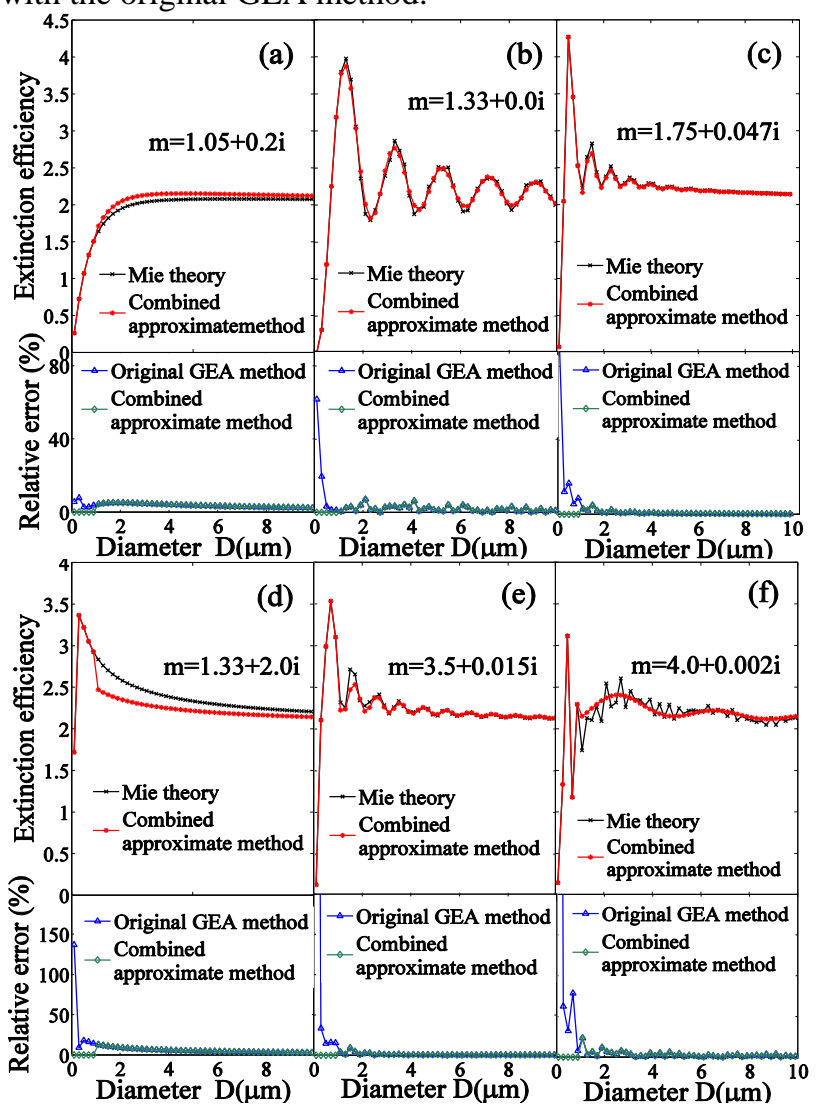

Fig. 1. Comparison of extinction efficiency of some common and extreme particles by the exact Mie theory and combined approximation method at $\lambda=0.6328 \mu \mathrm{m}$.

For common particles, the extinction efficiency calculated by the combined approximate method can fit the exact Mie extinction efficiency everywhere in the whole diameter region. When an imaginary part of the refractive index is relatively larger [Fig. 1(d)], because of less oscillation characteristics, smaller differences between different wavelengths makes it hard to retrieve PSD in the spectral extinction technique. When the real part of the refractive index is larger [Fig. 1(f)], the ripple structures become much more pronounced and there are larger discrepancies. To guarantee the accuracy for subsequent retrieval of PSD, the range of the refractive index in this paper is limited to $1.0 \leq m_{r} \leq 3.5,0 \leq m_{i} \leq 2.0$, which basically covers the most common natural and artificial particles.

The main objective of this paper is to show that the combined approximate method can be alternatively applied to retrieval of PSD in the independent model. We do not address the comparison of different inversion methods. Here, we adopt the combination of the Philips-Twomey regularization and non-negative least squares (PT-NNLS) approach as the inversion method [8].
The retrieved PSD function $f(D)$ can be given as

$$
f=\left(A^{T} A+\gamma H\right)^{-1} A^{T} E
$$

where $E=\left[E_{1}, E_{2}, \cdots E_{s}\right]^{T}, E_{s}=\ln \left[I\left(\lambda_{s}\right) / I_{0}\left(\lambda_{s}\right)\right] ; A$ is the weigh matrix about extinction efficiency calculated by the proposed method; $H$ is the covariance matrix. The regularization parameter $\gamma$ can be estimated by the Generalized Cross-Validation (GCV) technique which meets the minimization of the following objective function:

$$
V(\gamma)=\frac{\frac{1}{M}\|[I-K(\gamma)] E\|_{2}^{2}}{\left\{\frac{1}{M} \operatorname{trace}[I-K(\gamma)]\right\}^{2}}
$$

where $K(\gamma)=A\left(A^{T} A+\gamma H\right)^{-1} A^{T}$ is the influence matrix and $I$ is the identity matrix.

First, the validity of the method in retrieving PSD is verified through computer simulation. The diameter $D$ is limited in the range of $0.1-10.0 \mu \mathrm{m}$. The unimodal and bimodal Rosin-Rammler (R-R) functions are used as the preset PSD functions, which can be defined as:

$$
\begin{gathered}
f(D)_{u}=\frac{k}{\bar{D}} \times\left(\frac{D}{\bar{D}}\right)^{k-1} \times \exp \left[-\left(\frac{D}{\bar{D}}\right)^{k}\right] \\
f(D)_{b}=n\left[\frac{k_{1}}{\bar{D}_{1}} \times\left(\frac{D}{\bar{D}_{1}}\right)^{k_{1}-1} \times \exp \left(-\left(\frac{D}{\bar{D}_{1}}\right)^{k_{1}}\right)\right] \\
+(1-n)\left[\frac{k_{2}}{\bar{D}_{2}} \times\left(\frac{D}{\bar{D}_{2}}\right)^{k_{2}-1} \times \exp \left(-\left(\frac{D}{\bar{D}_{2}}\right)^{k_{2}}\right)\right]
\end{gathered}
$$

where $\bar{D}, k, \overline{D_{1}}, k_{1}, \overline{D_{2}}, k_{2}, n$ are the characteristic parameters, $0 \leq n \leq 1$.

Figure 2 shows the retrieval results of narrow and broad unimodal band distribution. 17 wavelengths of $0.4-2.0 \mu \mathrm{m}$ are randomly selected in the visible-near-infrared spectrum region. Numerical integration is carried out using a simple trapezoidal quadrature method and the discretization step is $0.2 \mu \mathrm{m}$. Fig. 3 shows the retrieval results of bimodal R-R distribution. The discretization step is still $0.2 \mu \mathrm{m}$ but 23 wavelengths are selected in this case.
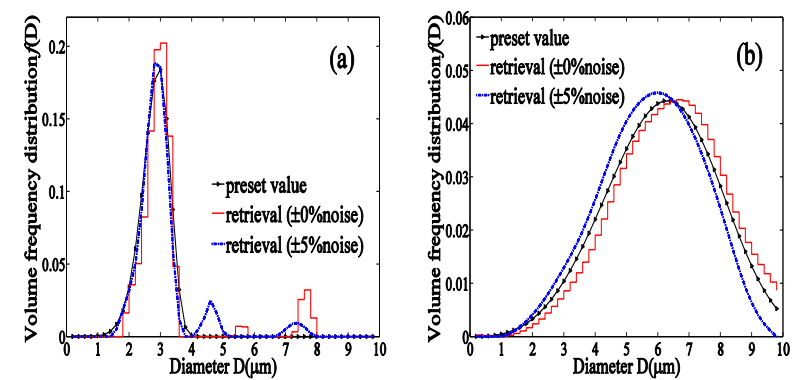

Fig. 2. Retrieval results of unimodal R-R distribution in the framework of approximate $\operatorname{method}(m=1.33+0 \mathrm{i}):(\mathrm{a})(\bar{D}, k)=(3.0,7.5)(\mathrm{b})$

$(\bar{D}, k)=(6.8,3.9)$.

In these figures, the retrieval results for a narrow and broad band and bimodal distribution, whose peaks differ evidently in diameter [Fig. 3(a)], can be considered satisfactorily, despite weak artifact peaks and a slight 
tendency for tails in Fig. 2(a) and Fig. 3(a). While for the bimodal distribution whose peaks are similar in diameter [Fig. 3 (b)], the positions of two peaks can be reconstructed correctly. But there are large discrepancies in height and width of the two peaks. Meanwhile, the third artifact peak appears with $3 \%$ random noise added.
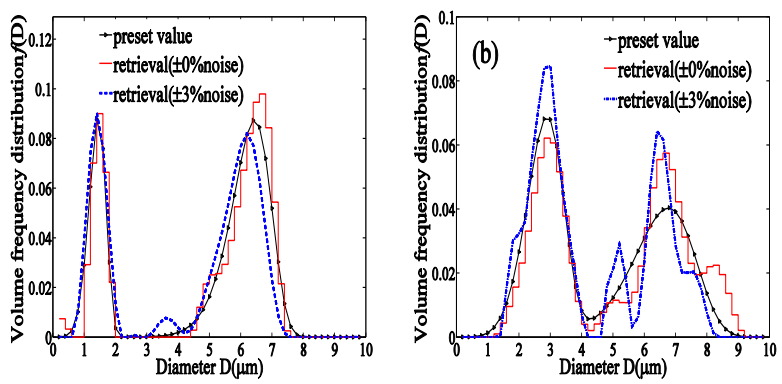

Fig. 3. Retrieval results of bimodal R-R distribution by using approximate method $(m=1.33+0 \mathrm{i})$.

(a) $\left(\bar{D}_{1}, k_{1}, \bar{D}_{2}, k_{2}, n\right)=(1.5,6.0,6.5,11.0,0.3)$

(b) $\left(\left(\bar{D}_{1}, k_{1}, \bar{D}_{2}, k_{2}, n\right)\right)=(3.0,5.5,6.9,7.5,0.5)$.

We also examine the retrieval time on an Intel Pentium $2.8 \mathrm{GHz}$ PC. The whole retrieval process of Fig. 2, for example, by using the combined approximate method consumes about 1.6s. Under the same computational conditions, the retrieval time by using the Mie theory is about $74.7 \mathrm{~s}$. The retrieval time by using the combined approximate method is significantly reduced compared with the Mie theory. The savings in time and memory resources are important for on-line measurement.

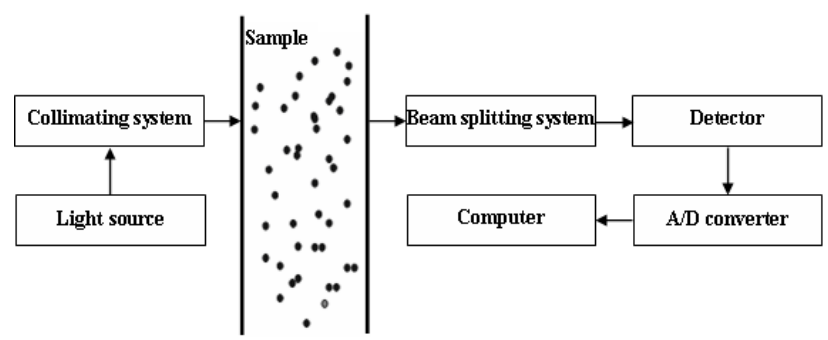

Fig. 4. Schematic of measurement system.

Last, the validity of the method in retrieving PSD is further verified through reference material. The schematic of the measurement system is shown in Fig. 4. A halogen lamp is used as a light source which gives a spectrum from 0.3 to $2.4 \mu \mathrm{m}$. By means of a beam splitting system, nine wavelengths in the range of $0.38-0.78 \mu \mathrm{m}$ are picked out from the light source and used to make extinction measurements. The experiment for each wavelength consists of two steps. The first step is to detect the spectrum of transmitted light intensity in absence of particles, and the second step is to detect the spectrum of transmitted light intensity with the particles filled. The extinction values of different wavelengths are obtained by comparing the spectrum of the two steps. To prevent the sedimentation of particles in a suspension, a stirrer is also used.
The standard monodisperse spherical polystyrene samples with labeled diameter $D=1.98 \mu \mathrm{m}$ are dispersed in water whose relative refractive index is $m=1.235$. Relevant experimental results are shown in Fig. 5. The Sauter mean diameters $D_{32}$ obtained by the two methods are $2.029 \mu \mathrm{m}$ and $2.058 \mu \mathrm{m}$, respectively. The relative errors with the labeled value are $6.9 \%$ and $7.3 \%$, respectively. The diameters retrieved by the two methods are all close to the labeled one. Discrepancies of the diameter satisfy the demand of standard monodisperse polystyrene particles.

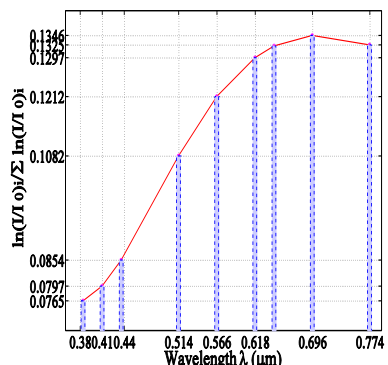

(a)

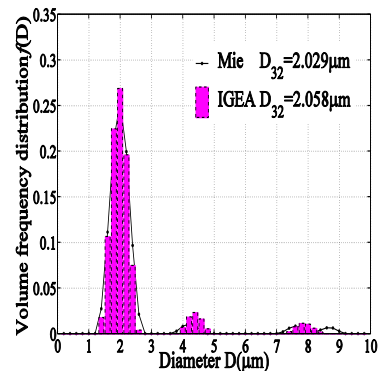

(b)
Fig. 5. Relevant experimental results: (a) relationship of incident wavelength and light intensity, and (b) retrieval results of standard polystyrene particle in the framework of Mie theory and approximate method.

In summary, the combined approximate method can be used as an alternative to the rigorous Mie theory for retrieval of PSD in the independent model. Within the framework of a combined approximate method, the retrieval time can be significantly reduced. The reliability and rapidity of retrieval is important for on-line measurement. There is still a lot of work to be done. The approximation method for fast retrieval of non-spherical particle size distribution will be investigated in further studies.

This work was supported by the National Natural Science Foundation of China (No. 61071036). The authors are grateful to Prof. X. S. Cai (Institute of Particle and Two Phases Flow Measurement, University of Shanghai for Science \& Technology) for the offer of experimental data.

\section{References}

[1] C.F. Bohren, D.R. Huffman, Absorption and Scattering of Light by Small Particles (Wiley, 1998).

[2] H.Y. Zuo et al., Opt. Lett. 35, 1380 (2010).

[3] M.Z. Li, D. Wilkinson, Chem. Eng. Sci. 56, 3045 (2001).

[4] X.Z. Li et al., Appl. Opt. 46, 5241 (2007).

[5] X.G. Sun, H. Tang, J.M. Dai, Opt. Exp. 15, 11507 (2007).

[6] S.K. Sharma, D.J. Somerford, Light Scattering by Optically Soft Particles: Theory and Applications (Springer-Praxis, 2006).

[7] T.W. Chen, Appl. Opt. 28, 4096 (1989).

[8] M. Kandlikar, G. Ramachandran, J. Aerosol Sci. 30, 413 (1999). 